\title{
Applications of Driftless Control Affine Systems to a Problem of Inventory and Production
}

\author{
Liviu Popescu \\ Department of Statistics and Economic Informatics, Faculty of Economics and Business Administration, \\ University of Craiova, 13 Al. I. Cuza Street, Craiova 200585, Romania \\ liviunew@yahoo.com; liviupopescu@central.ucv.ro
}

\begin{abstract}
In this paper, the framework of driftless control affine systems has been used for the study of inventory and production problems. A mathematical model for an economic problem was built by using some optimal control techniques. For the controllability issue of the optimal control system, which involves restrictions on the final quantities required, the Lie geometric methods have been applied. In order to find the optimal solution, the Pontryagin Maximum Principle has been used at the level of a Lie algebroid, which in this case consists in an integrable distribution of the tangent space. Finally, a numerical example is presented.
\end{abstract}

Keywords: Optimal control, Pontryagin Maximum Principle, Inventory and production, Control affine systems, Lie algebra.

\section{Introduction}

It is well known that the optimal control theory has been applied successfully in different domains such as economics, econometrics, cybernetics or operations research. Arrow (1968) applied the control theory to economic growth. The book of Seierstad \& Sydsater (1987) added a valuable contribution to the literature on the optimal control of economic processes. Sethi \& Thompson (2000) covered topics including production and inventory problems, optimal consumption of natural resources or applications of control theory to economics. Caputo (2005) presented numerous complementary methods for applications of optimal control to operations research and management science. Weber (2011) provided an introduction to the use of optimal control techniques for deterministic continuoustime systems in economics.

The Lie geometric methods are used in the study of controllability problems of control systems by many authors. Brocket (1973) proved that Lie theory and control are closely connected. In the book of Isidori (1995) the control affine systems are studied. LaValle (2006) presented a unified treatment of control theory including holonomic and nonholonomic systems. Some type of control mechanical systems are investigated by Popescu (2018) or Khan, Akmeliawati \& Khan (2018).

One of the most important and powerful methods in the study and analysis of the solutions for the optimal control problem is Pontryagin's Maximum Principle, which generates a first order necessary condition for optimality. It is assumed that a curve $c(t)=(x(t) u(t)$ is an optimal solution if there exists a lifting of $x(t)$ to the cotangent space $(x(t) p(t)$ satisfying the Hamilton-
Jacobi-Bellman equations. However, finding the optimal solution for a control problem remains very difficult for several reasons. First of all, the problem of integrating a Hamiltonian system, which is generally difficult to integrate, except for its particular dynamics and costs, is approached. Secondly, even if all solutions can be found, the problem is to select the optimal trajectories from among them. For these reasons, it is very important to find new methods and techniques that would simplify this study. The aim of this paper is to solve a problem pertaining to inventory and production. This type of problems is intensely studied. Ortega \& Lin (2004) gave a review of control theory applications to the productioninventory problem. A study on supply planning and inventory control under lead time uncertainty is presented by Dolgui et al. (2013). Gayon, Vercraene \& Flapper (2017) studied optimal control of a production-inventory system with product returns. A problem of production control under inventory inaccuracy and time-delay is investigated by Li \& Wang (2017). Chen (2018) studied a problem for optimization of production inventory for a single-vendor multi-buyer system of perishable products.

In this paper the Pontryagin Maximum Principle is used at the level of a new working space. The fact that the framework of Lie algebroids is more suitable than the cotangent space for the study of driftless control affine systems with holonomic distribution is proven. A Lie algebroid is a generalization of the notions of tangent space and Lie algebra. The symmetries of control systems using the framework of Lie algebroids are studied by Popescu (2017). This paper is 
organized as follows. In the second section, some necessary results from optimal control theory are introduced. Also, the control affine systems are presented and the problem of controllability is studied, by using the properties of Lie brackets in the case of holonomic distributions. Next, only the necessary notions about Lie algebroids and the geometric viewpoint of the optimal control in this new framework are presented. Finally, the relation between the Hamiltonian function $\widetilde{H}$ on dual Lie algebroid and the Hamiltonian function $H$ on the cotangent space is given.

In section three, that contains the novelty of the paper, the strategy is to apply the Pontryagin Maximum Principle at the level of a Lie algebroid for a problem of inventory and production. First, we present the mathematical model of our problem and observe that this is a driftless control affine system with holonomic distribution. Using the Lie brackets of the vectors of distribution and the Frobenius theorem we study the controllability and find the condition that the inventory level has to fulfill, such that the system can be brought from an initial state to a final state. In order to find the optimal solution, we can apply the Pontryagin Maximum Principle as in the classical case on the cotangent space, but we obtain a very difficult system of differential equations. We will use a different approach, involving the framework of Lie algebroids. Using the HamiltonJacobi-Bellman equations on Lie algebroids we find the solution, which is optimal because the Hamiltonian function is convex.

\section{Optimal Control}

Let $M$ be a smooth $n$-dimensional manifold (in particular, a subset of $R^{n}$ ). A control system on $M$ given by a set of differential equations of the form

$$
\frac{d x^{i}}{d t}=f^{i}(x, u)
$$

will be considered, where $x=\left(x^{1}, \ldots, x^{n}\right) \in M$ represents the state variables of the system and $u=\left(u^{1}, \ldots, u^{m}\right) \in U \subset R^{m}$ represents its control variables. Let $x_{0}$ and $x_{1}$ be two states of the system (two points of $M$ ). An optimal control problem consists in finding the ways in which the system is brought from the initial state $x_{0}$ to the final state $x_{1}$ and in minimizing the cost functional

$$
\min _{u} \int_{0}^{T} L(x(t), u(t)) d t, x(0)=x_{0}, x(T)=x_{1},
$$

where L is the Lagrangian (energy, cost, time, distance, etc.).

In other words, the trajectories of our control system which connects two points have to be found, so that a certain optimality condition is satisfied. The necessary condition for a solution to be optimal is given by the Pontryagin Maximum Principle (Pontryagin, 1962). The Hamiltonian function on dual space is given by $H=\langle p, f\rangle-L$, which leads to

$H(x, p, u)=\sum_{i=1}^{n} p_{i} f^{i}(x, u)-L(x, u)$,

where $\left(p_{1}, \ldots, p_{n}\right)$ are momentum variables. The maximization condition with respect to control variables $u$,

$H(x(t), p(t), u(t))=\max _{v} H(x(t), p(t), v)$,

yields

$\frac{\partial H}{\partial u^{i}}=0$

( $H$ is assumed to be smooth with respect to $u$ ) and the extreme trajectories satisfy the equations

$\frac{d x^{i}}{d t}=\frac{\partial H}{\partial p_{i}}, \quad \frac{d p_{i}}{d t}=-\frac{\partial H}{\partial x^{i}}$

\subsection{Control Affine Systems}

A control affine system has the following form (LaValle, 2006)

$\dot{x}=X_{0}(x)+\sum_{i=1}^{m} u^{i} X_{i}(x)$

where $\dot{x}=\frac{d x}{d t}$ and $X_{0}, X_{1}, \ldots, X_{m}$ are smooth vector fields on the manifold $M$. Usually, $X_{0}$ is called the drift vector field as it describes the dynamics of the system in the absence of controls and $X_{i}, i=\overline{1, m}$ are called input vector fields. A first aspect studied for these systems is the issue of controllability.

A system is deemed controllable if for any two states $x_{0}$ and $x_{1}$ there exists a solution curve of (2) connecting $x_{0}$ to $x_{1}$. Controllability does not depend on the quality of the trajectory between two states, or on the amount of control effort. The presence of drift complicates the issue of controllability significantly. Further on, a driftless control affine system, or a distributional system, 
where $X_{0}=0$, will be considered. In this case the following can be obtained:

$\dot{x}=\sum_{i=1}^{m} u^{i} X_{i}(x)$

Most information about controllability is included in the structure of the Lie algebra generated by the vector fields $X_{i}, i=\overline{1, m}$. A distribution $\Delta$ on the manifold $M$ is a map which assigns to each point in $M$ a subspace of the tangent space for the respective point, namely $x \in M \rightarrow \Delta(x) \subset T_{x} M$.

The distribution $\Delta$ is considered locally finitely generated if there is a family of vector fields $X_{i}$, $i=\overline{1, m}$ (called local generators of $\Delta$ ) which spans $\Delta$, i.e.

$$
\Delta(x)=\operatorname{span}\left\{X_{1}(x), \ldots, X_{m}(x)\right\} \subset T_{x} M
$$

The distribution $\Delta$ has the dimension $k$ if $\operatorname{dim} \Delta(x)=k$, for all points $x \in M$. In this regard, the Lie bracket of two vector fields is given by

$$
[X, Y](f)=X(Y(f))-Y(X(f)) .
$$

Let $\Delta=\operatorname{span}\left\{X_{1}, \ldots, X_{m}\right\}$ be the distribution generated by the family of vector fields $X_{i}$, $i=1, m$. The distribution is called involutive if the Lie bracket of any two vectors from $\Delta$ belongs to $\Delta$, that is if

$$
X, Y \in \Delta \Rightarrow[X, Y] \in \Delta .
$$

In this case every Lie bracket can be expressed as a linear combination of the system vector fields, and therefore

$$
\left[X_{i}, X_{j}\right]=\sum_{k=1}^{m} L_{i j}^{k} X_{k}
$$

It should be mentioned that a foliation $\left\{S_{\alpha}\right\}_{\alpha \in I}$ of $M$ is a partition of $M=\bigcup_{\alpha \in I} S_{\alpha}$ into disjoint connected submanifolds $S_{\alpha}$ called leaves.

Definition 1. A distribution $\Delta$ of constant dimension on $M$ is called integrable (holonomic) if there exists a foliation $\left\{S_{\alpha}\right\}_{\alpha \in I}$ on $M$ whose tangent bundle is $\Delta$, that is $T_{x} S=\Delta(x)$, where $S$ is the leaf passing through $x$.

Theorem 1. (Frobenius) If $\Delta$ is a distribution with constant dimension on the manifold $M$, then $\Delta$ is integrable if and only if $\Delta$ is involutive.

If this is applied in the case of driftless control affine system (3) and the distribution $\Delta$, generated by the input vectors $X_{i}, i=\overline{1, m}$ is integrable (holonomic) with constant dimension, then the system is not controllable and $\Delta$ determines a foliation on $M$ with the property that any curve is contained in a single leaf of the foliation. In other words, any two points can be joined by an optimal trajectory if and only if they are situated on the same leaf.

Further on, some notions about Lie algebroids, which are useful in the study of driftless control affine systems will be presented.

\subsection{Lie Algebroids}

Let $M$ be a real, $C^{\infty}$-differentiable, $n$-dimensional manifold and $T_{x} M$ its tangent space at $x \in M$. The tangent space of $M$ is $\left(T M, \pi_{M}, M\right)$ where $T M=\bigcup_{x \in M} T_{x} M$ and $\pi_{M}$ is the canonical projection map $\pi_{M}: T M \rightarrow M$ taking a tangent vector $X(x) \in T_{x} M \subset T M$ to the base point $x \in M$. A vector bundle is a triple $(E, \pi, M)$ where $E$ and $M$ are manifolds, called the total space and the base space and the map $\pi: E \rightarrow M$ is a surjective submersion. According to Mackenzie (1987), the following can be obtained:

Definition 2. A Lie algebroid over the manifold $M$ is a triple $\left(E,[\cdot, \cdot]_{E}, \sigma\right)$ where $(E, \pi, M)$ is a vector bundle of rank $m$ over $M$, satisfying the following conditions:

a) $C^{\infty}(M)$-module of sections of $\Gamma(E)$ is endowed with a Lie algebra structure $[\because,]_{E}$.

b) $\sigma: E \rightarrow T M$ is a bundle map, usually called the anchor, which induces a Lie algebra homomorphism from the Lie algebra of sections of the bundle $\left(\Gamma(E)[\because,]_{E}\right)$ to the Lie algebra of vector fields $(\Gamma(M),[\because]$, thereby satisfying the Leibniz rule

$$
\begin{aligned}
& {\left[s_{1}, f s_{2}\right]_{E}=f\left[s_{1}, s_{2}\right]_{E}+\left(\sigma\left(s_{1}\right) f\right) s_{2},} \\
& \forall s_{1}, s_{2} \in \Gamma(E) \quad f \in C^{\infty}(M)
\end{aligned}
$$

From this definition it results that:

1. $[\because,]_{E}$ is a $R$-bilinear operation.

2. $[\because,]_{E}$ is skew-symmetric, i.e. $\left[s_{1}, s_{2}\right]_{E}=-\left[s_{2}, s_{1}\right]_{E}$.

3. $[\because,]_{E}$ verifies the Jacobi identity,

$$
\begin{aligned}
& {\left[s_{1},\left[s_{2}, s_{3}\right]_{E}\right]_{E}+\left[s_{2},\left[s_{3}, s_{1}\right]_{E}\right]_{E}} \\
& +\left[s_{3},\left[s_{1}, s_{2}\right]_{E}\right]_{E}=0 .
\end{aligned}
$$


For a function $f$ on $M$, the differential $d f(x) \in E_{x}^{*}$ is given by $\langle d f(x), a\rangle=\sigma(a) f$ for $\forall a \in E_{x}$. If $\omega \in \wedge^{k}\left(E^{*}\right)$ is a $k$-form, then the exterior derivative $d^{E} \omega \in \wedge^{k+1}\left(E^{*}\right)$ is given by

$$
\begin{aligned}
& d^{E} \omega\left(s_{1}, \ldots, s_{k+1}\right)= \\
& \sum_{i=1}^{k+1}(-1)^{i+1} \sigma\left(s_{i}\right) \omega\left(s_{1}, \ldots, \widehat{s}_{i}, \ldots, s_{k+1}\right) \\
& +\sum_{1 \leq i<j \leq k+1}(-1)^{i+j} \omega\left(\left[s_{i}, s_{j}\right]_{E}, s_{1}, \ldots, \widehat{s}_{i}, \ldots, \widehat{s}_{j}, \ldots, s_{k+1}\right)
\end{aligned}
$$

where $s_{i} \in \Gamma(E)$. If the local coordinates $\left(x^{i}\right)$ are taken on an open $U \subset M$, then the local basis $\left\{s_{\alpha}\right\}$ of the bundle sections generates the local functions $\sigma_{i}^{\alpha}(x) L_{\alpha \beta}^{\gamma}(x)$ on M given by

$$
\begin{aligned}
\sigma\left(s_{\alpha}\right) & =\sigma_{i}^{\alpha} \frac{\partial}{\partial x^{i}}, \quad\left[s_{\alpha}, s_{\beta}\right]=L_{\alpha \beta}^{\gamma} s_{\gamma}, \quad i=\overline{1, n} \\
\alpha, \beta, \gamma & =\overline{1, m}
\end{aligned}
$$

which are called the structure functions of Lie algebroid.

A control system on the Lie algebroid $\left(E,[\cdot, \cdot]_{E}, \sigma\right)$ (Martinez, 2004) with the control space $\tau$ : $A \rightarrow M$ is given by a section $\rho$ of $E$ along $\tau$. A trajectory of the control system $\rho$ is an integral curve of the vector field $\sigma(\rho)$. In relation to a cost function $\widetilde{L} \in C^{\infty}(A)$ the integral of $\widetilde{L}$ should be minimized over the set of those system trajectories which satisfy certain optimal conditions. The Hamiltonian function $\widetilde{H}$ is defined by

$\widetilde{H}(\mu, u)=\langle\mu, \rho(u)\rangle-\widetilde{L}(u)$,

whereas the associated Hamiltonian control system $\rho_{\widetilde{H}}$ is given by the symplectic equation of Lie algebroids

$i_{\rho_{\widetilde{H}}} \omega_{E}=d^{E} \widetilde{H}$,

where $\omega_{E}$ is the canonical symplectic form. The extremal trajectories are given by the HamiltonJacobi equations (Martinez, 2004)

$\frac{d x^{i}}{d t}=\sigma_{\alpha}^{i} \frac{\partial \widetilde{H}}{\partial \mu_{\alpha}}$,

$\frac{d \mu_{\alpha}}{d t}=-\sigma_{\alpha}^{i} \frac{\partial \widetilde{H}}{\partial x^{i}}-\mu_{\gamma} L_{\alpha \beta}^{\gamma} \frac{\partial \widetilde{H}}{\partial \mu_{\beta}}$

with $\frac{\partial \widetilde{H}}{\partial u^{A}}=0$.
We can associate to any Lagrangian $\widetilde{L}: E \rightarrow R$ on Lie algebroid $E$, a Lagrangian $L$ on $\operatorname{Im} \sigma \subset T M$ according to (Popescu, 2009)

$$
L(v)=\left\{\widetilde{L}(u) \mid u \in E_{x}, \quad \sigma(u)=v\right\} .
$$

Theorem 2. The connection between the Hamiltonian function $H$ on dual space $T^{*} M$ and the Hamiltonian function $\widetilde{H}$ on the dual Lie algebroid $E^{*}$ is given by

$$
\begin{gathered}
H(p)=\widetilde{H}\left(\sigma^{*}(p)\right), \quad \mu=\sigma^{*}(p) \\
p \in T_{x}^{*} M, \quad \mu \in E_{x}^{*}
\end{gathered}
$$

Proof. The Fenchel-Legendre dual of Lagrangian function $L$ is the Hamiltonian function $H$ given by

$H(p)=\sup _{v}\{\langle p, v\rangle-L(v)\}$

$=\sup _{v}\{\langle p, v\rangle-\widetilde{L}(u), \quad \sigma(u)=v\}$

and the following is obtained

$$
\begin{aligned}
& H(p)=\sup _{u}\{\langle p, \sigma(u)\rangle-\widetilde{L}(u)\} \\
& =\sup _{u}\left\{\left\langle\sigma^{*}(p), u\right\rangle-\widetilde{L}(u)\right\}=\widetilde{H}\left(\sigma^{*}(p)\right)
\end{aligned}
$$

where $\sigma^{*}: T^{*} M \rightarrow E^{*}$ is the dual application. In local coordinates

$\mu_{\alpha}=\sigma_{\alpha i}^{* i} p_{i}$

is obtained and the Hamiltonian function $H$ is degenerate on $\operatorname{Ker} \sigma^{*} \subset T^{*} M$.

\section{Applications of Control Affine Systems to a Problem of Inventory and Production}

It is assumed that a company manufactures three types of products, denoted by $P_{1}, P_{2}, P_{3}$. In a certain period of time $T$, which is fixed, it must produce a certain amount $\left(s_{1}, s_{2}, s_{3}\right)$ of each type of product. A certain quantity of the $P_{1}, P_{2}$ products are used for manufacturing the $P_{3}$ product. Also, it is known that the unit production costs increase linearly with the production level and the cost of production operations for $P_{3}$ is considered negligible. The unit storage costs for each product per unit time are given by constants $\left(\beta_{1}, \beta_{2}, \beta_{3}\right)$. A production plan is necessary to ensure the required quantity is produced until the specified delivery 
date with minimum costs.

The case of a single product is studied by Kamien \& Schwartz (2006).

Let us consider $x^{i}=x^{i}(t), i=1,2,3$ the inventory at time $t$. The inventory level is the cumulated past production $p^{i}=p^{i}(t)$, and considering $x^{i}(0)=0$, the following is obtained:

$x^{i}(t)=\int_{0}^{t} p^{i}(y) d y$

It results that the rate of change of the inventory $\dot{x}^{i}$ is the production and we have $\dot{x}^{i}=p^{i}$. As the unit production costs, denoted by $c_{i}$, increase linearly with the production level, $c_{i}=\alpha_{i} p^{i}$, the total cost of production is given by

$c_{1} p^{1}+c_{2} p^{2}=\alpha_{1}\left(p^{1}\right)^{2}+\alpha_{2}\left(p^{2}\right)^{2}=\alpha_{1}\left(\dot{x}^{1}\right)^{2}+\alpha_{1}\left(\dot{x}^{2}\right)^{2}$

where $\dot{a}_{1}, \dot{a}_{2}$ are positive constants. Thus, the total cost, including the costs of inventory holding is given by

$\alpha_{1}\left(\dot{x}^{1}\right)^{2}+\alpha_{2}\left(\dot{x}^{2}\right)^{2}+\beta_{1}\left(1-k_{1}\right) x^{1}$

$+\beta_{2}\left(1-k_{2}\right) x^{2}+\beta_{3} x^{3}$

where $k_{1}, k_{2} \in[0,1]$ represent the percentages of quantities $P_{1}, P_{2}$ used for the manufacturing of $P_{3}$. If it is considered that $\dot{x}^{1}=u^{1}, \dot{x}^{2}=u^{2}$ are the control variables and if it is assumed that the rate of change of the inventory level for $P_{3}$ is given by the law $\dot{x}^{3}=k_{1} u^{1} x^{1}+k_{2} u^{2} x^{2}$, the following optimal control problem is obtained:

$$
\left\{\begin{array}{c}
\dot{x}^{1}=u^{1} \\
\dot{x}^{2}=u^{2} \\
\dot{x}^{3}=k_{1} u^{1} x^{1}+k_{2} u^{2} x^{2} \\
x^{i}(0)=0, \quad x^{i}(T)=s_{i} \\
u^{1}, u^{2} \geq 0, \quad k_{1}, k_{2} \in[0,1]
\end{array}\right.
$$

A production plan with minimum costs is the solution

$$
\begin{aligned}
\min _{u} & \int_{0}^{T}\left(\alpha_{1}\left(u^{1}\right)^{2}+\alpha_{2}\left(u^{2}\right)^{2}\right. \\
& \left.+\beta_{1}\left(1-k_{1}\right) x^{1}+\beta_{2}\left(1-k_{2}\right) x^{2}+\beta_{3} x^{3}\right) d t
\end{aligned}
$$

It can be noticed that this is a driftless control affine system on $M=R_{+}^{3}$ written in the form $\dot{x}=u_{1} X_{1}+u_{2} X_{2}, \quad x=\left(x^{1}, x^{2}, x^{3}\right)^{t} \in R_{+}^{3}$

$\min _{u} \int_{0}^{T} F(u(t), x(t)) d t$,

where

$X_{1}=\left(\begin{array}{c}1 \\ 0 \\ k_{1} x^{1}\end{array}\right) \quad X_{2}=\left(\begin{array}{c}0 \\ 1 \\ k_{2} x^{2}\end{array}\right)$ and

$F(u(t), x(t))=\alpha_{1}\left(u^{1}\right)^{2}+\alpha_{2}\left(u^{2}\right)^{2}+\beta_{1}\left(1-k_{1}\right) x^{1}$

$+\beta_{2}\left(1-k_{2}\right) x^{2}+\beta_{3} x^{3}$

The optimal trajectories starting from the point $(0,0,0)$ to endpoint $\left(s_{1}, s_{2}, s_{3}\right)$ are sought. The distribution $\Delta=\operatorname{span}\left\{X_{1}, X_{2}\right\}$ generated by the vector fields $X_{1}, X_{2}$ has a constant dimension, $\operatorname{dim} \Delta(x)=2$, for all $x \in R^{3}$. Also, in the natural basis $\left\{\frac{\partial}{\partial x^{1}}, \frac{\partial}{\partial x^{2}}, \frac{\partial}{\partial x^{3}}\right\}$ of $R^{3}$ the vector fields have the expressions

$X_{1}=\frac{\partial}{\partial x^{1}}+k_{1} x^{1} \frac{\partial}{\partial x^{3}}, X_{2}=\frac{\partial}{\partial x^{2}}+k_{2} x^{2} \frac{\partial}{\partial x^{3}}$,

and by using the formula

$[f X, g Y]=f g[X, Y]+f X(g) Y-g Y(f) X$

it can be noticed that the Lie bracket is given by

$\left[X_{1}, X_{2}\right]=\left[\frac{\partial}{\partial x^{1}}+k_{1} x^{1} \frac{\partial}{\partial x^{3}}, \frac{\partial}{\partial x^{2}}+k_{2} x^{2} \frac{\partial}{\partial x^{3}}\right]=0$,

that is the distribution $\Delta$ is involutive. From the Frobenius theorem, it results that the distribution is integrable (holonomic) and that it determines a foliation on $R_{+}^{3}$ and two points can be joined by an optimal trajectory if and only if they are situated on the same leaf. In fact, the economic system is not controllable, in the sense that we cannot reach any final stock quantity. Indeed, from the system (7) the following can be obtained

$\dot{x}^{3}=k_{1} \dot{x}^{1} x^{1}+k_{2} \dot{x}^{2} x^{2}$,

and it results that

$x^{3}=\frac{k_{1}\left(x^{1}\right)^{2}+k_{2}\left(x^{2}\right)^{2}}{2}+c, \quad c \in R$

which are the surfaces (elliptic paraboloids) of $R_{+}^{3}$ , which determine a foliation. Moreover, by using $x^{i}(0)=0$ the following relation can be obtained:

$x^{3}=\frac{k_{1}\left(x^{1}\right)^{2}+k_{2}\left(x^{2}\right)^{2}}{2}$, 
and from $x^{i}(T)=s_{i}$ it results that the abovementioned problem has a solution curve connecting $(0,0,0)$ to endpoint $\left(s_{1}, s_{2}, s_{3}\right)$ (the system is controllable) if and only if the final amounts satisfy the condition

$s_{3}=\frac{k_{1} s_{1}^{2}+k_{2} s_{2}^{2}}{2}$.

Next, the Pontryagin Maximum Principle can be used in order to find the optimal solution. The Hamiltonian function on dual space is given by

$H=\sum_{i=1}^{3} p_{i} \dot{x}^{i}-F$ which leads to

$H=p_{1} u^{1}+p_{2} u^{2}+p_{3}\left(k_{1} u^{1} x^{1}+k_{2} u^{2} x^{2}\right)$

$-\alpha_{1}\left(u^{1}\right)^{2}-\alpha_{2}\left(u^{2}\right)^{2}-\beta_{1}\left(1-k_{1}\right) x^{1}$

$-\beta_{2}\left(1-k_{2}\right) x^{2}-\beta_{3} x^{3}$,

where $p_{1}, p_{2}, p_{3}$ are momentum variables on dual space. The condition $\frac{\partial H}{\partial u}=0$ leads to the following equations

$\frac{\partial H}{\partial u^{1}}=0 \Rightarrow u^{1}=\frac{p_{1}+p_{3} k_{1} x^{1}}{2 \alpha_{1}}$

$\frac{\partial H}{\partial u^{2}}=0 \Rightarrow u^{2}=\frac{p_{2}+p_{3} k_{2} x^{2}}{2 \alpha_{2}}$

Now, the expressions of the control variables $u^{1}, u^{2}$ will be replaced into the expression of the Hamiltonian function and by direct computation it results that

$H=\frac{\left(p_{1}+p_{3} k_{1} x^{1}\right)^{2}}{4 \alpha_{1}}+\frac{\left(p_{2}+p_{3} k_{2} x^{2}\right)^{2}}{4 \alpha_{2}}$

$-\beta_{1}\left(1-k_{1}\right) x^{1}-\beta_{2}\left(1-k_{2}\right) x^{2}-\beta_{3} x^{3}$

Using the Hamilton-Jacobi-Bellman equations (1) the following can be obtained:

$\dot{x}^{1}=\frac{\partial H}{\partial p_{1}}=\frac{p_{1}+p_{3} k_{1} x^{1}}{2 \alpha_{1}}$,

$\dot{x}^{2}=\frac{\partial H}{\partial p_{2}}=\frac{p_{2}+p_{3} k_{2} x^{2}}{2 \alpha_{2}}$,

$\dot{x}^{3}=\frac{\partial H}{\partial p_{3}}=\frac{\left(p_{1}+p_{3} k_{1} x^{1}\right) k_{1} x^{1}}{2 \alpha_{1}}+\frac{\left(p_{2}+p_{3} k_{2} x^{2}\right) k_{2} x^{2}}{2 \alpha_{2}}$

$\dot{p}_{1}=-\frac{\partial H}{\partial x^{1}}=\beta_{1}\left(1-k_{1}\right)-\frac{\left(p_{1}+p_{3} k_{1} x^{1}\right) k_{1} p_{3}}{2 \alpha_{1}}$, $\dot{p}_{2}=-\frac{\partial H}{\partial x^{2}}=\beta_{2}\left(1-k_{2}\right)-\frac{\left(p_{2}+p_{3} k_{2} x^{2}\right) k_{2} p_{3}}{2 \alpha_{2}}$,

$\dot{p}_{3}=-\frac{\partial H}{\partial x^{3}}=\beta_{3}$

which is a complicated system of differential equations.

In order to find the optimal solution, a different approach, which involves the framework of Lie algebroids, will be used. The Pontryagin Maximum Principle will be applied for the Lie algebroid $E=\Delta$ (holonomic distribution with constant rank), the state space is $M=R_{+}^{3}$, the anchor $\sigma: E \rightarrow T M$ is the inclusion and $[\cdot \cdot \cdot]$ the induced Lie bracket. In this case, the anchor $\sigma$ has the components

$\sigma_{\alpha}^{i}=\left(\begin{array}{cc}1 & 0 \\ 0 & 1 \\ k_{1} x^{1} & k_{2} x^{2}\end{array}\right), \quad i=\overline{1,3}, \quad \alpha=\overline{1,2}$

and a regular Lagrangian function on Lie algebroid $E$ will be obtained in the form

$\tilde{L}(u(t), x(t))=\alpha_{1}\left(u^{1}\right)^{2}+\alpha_{2}\left(u^{2}\right)^{2}+\beta_{1}\left(1-k_{1}\right) x^{1}$

$+\beta_{2}\left(1-k_{2}\right) x^{2}+\beta_{3} x^{3}$

Indeed,

$\frac{\partial^{2} \widetilde{L}}{\partial u^{i} \partial u^{j}}=\left(\begin{array}{cc}2 \alpha_{1} & 0 \\ 0 & 2 \alpha_{2}\end{array}\right)$

and it results that

$\operatorname{det}\left(\frac{\partial^{2} \widetilde{L}}{\partial u^{i} \partial u^{j}}\right) \neq 0$

Using the Legendre transformation induced by the regular Lagrangian

$(x, u) \rightarrow(x, \mu), \quad \mu_{i}=\Phi_{i}(x, u)=\frac{\partial \widetilde{L}}{\partial u^{i}}$

the Hamiltonian function is obtained:

$\widetilde{H}(x, \mu)=\mu \Phi^{-1}(x, \mu)-\widetilde{L}\left(x, \Phi^{-1}(x, \mu)\right)$

For the $(x, u)$ coordinates the following is obtained:

$\widetilde{H}=u^{i} \frac{\partial \widetilde{L}}{\partial u^{i}}-\widetilde{L}=\alpha_{1}\left(u^{1}\right)^{2}+\alpha_{2}\left(u^{2}\right)^{2}$

$-\beta_{1}\left(1-k_{1}\right) x^{1}-\beta_{2}\left(1-k_{2}\right) x^{2}-\beta_{3} x^{3}$

and the relations

$\mu_{1}=2 \alpha_{1} u^{1}, \quad \mu_{2}=2 \alpha_{2} u^{2}$ 
lead to the expression of the Hamiltonian function on the dual algebroid

$\widetilde{H}(x, \mu)=\frac{\mu_{1}^{2}}{4 \alpha_{1}}+\frac{\mu_{2}^{2}}{4 \alpha_{2}}-\beta_{1}\left(1-k_{1}\right) x^{1}$

$-\beta_{2}\left(1-k_{2}\right) x^{2}-\beta_{3} x^{3}$

Using the relation (6) the following expression can by obtained:

$\left(\begin{array}{l}\mu_{1} \\ \mu_{2}\end{array}\right)=\left(\begin{array}{lll}1 & 0 & k_{1} x^{1} \\ 0 & 1 & k_{2} x^{2}\end{array}\right)\left(\begin{array}{l}p_{1} \\ p_{2} \\ p_{3}\end{array}\right) \Rightarrow\left\{\begin{array}{l}\mu_{1}=p_{1}+k_{1} x^{1} p_{3} \\ \mu_{2}=p_{2}+k_{2} x^{2} p_{3}\end{array}\right.$

and from $H(p)=\widetilde{H}\left(\sigma^{*}(p)\right)$ it can be noticed that the expression of the Hamiltonian $\mathrm{H}$ on the cotangent bundle is the same as in (8). From the relation $\left[X_{\alpha}, X_{\beta}\right]=L_{\alpha \beta}^{\gamma} X_{\gamma}$ it can be obtained that $L_{\alpha \beta}^{\gamma}=0$ which leads to the Hamilton-Jacobi equations on the Lie algebroid (4) in the form

$\frac{d x^{i}}{d t}=\sigma_{\alpha}^{i} \frac{\partial \tilde{H}}{\partial \mu_{\alpha}}, \quad \frac{d \mu_{\alpha}}{d t}=-\sigma_{\alpha}^{i} \frac{\partial \tilde{H}}{\partial x^{i}}$

In this case the following system of differential equations is obtained:

$\dot{x}^{1}=\sigma_{1}^{1} \frac{\partial \widetilde{H}}{\partial \mu_{1}}=\frac{\mu_{1}}{2 \alpha_{1}}$

$\dot{x}^{2}=\sigma_{2}^{2} \frac{\partial \widetilde{H}}{\partial \mu_{2}}=\frac{\mu_{2}}{2 \alpha_{2}}$

$\dot{x}^{3}=\sigma_{1}^{3} \frac{\partial \widetilde{H}}{\partial \mu_{1}}+\sigma_{2}^{3} \frac{\partial \widetilde{H}}{\partial \mu_{2}}=\frac{\mu_{1} k_{1} x^{1}}{2 \alpha_{1}}+\frac{\mu_{2} k_{2} x^{2}}{2 \alpha_{2}}$,

$\dot{\mu}_{1}=-\sigma_{1}^{1} \frac{\partial \widetilde{H}}{\partial x^{1}}-\sigma_{1}^{3} \frac{\partial \widetilde{H}}{\partial x^{3}}=\beta_{1}\left(1-k_{1}\right)+k_{1} x^{1} \beta_{3}$,

$\dot{\mu}_{2}=-\sigma_{2}^{2} \frac{\partial \widetilde{H}}{\partial x^{2}}-\sigma_{2}^{3} \frac{\partial \tilde{H}}{\partial x^{3}}=\beta_{2}\left(1-k_{2}\right)+k_{2} x^{2} \beta_{3}$,

Moreover, from

$\dot{x}^{1}=\frac{\mu_{1}}{2 \alpha_{1}} \Rightarrow \ddot{x}^{1}=\frac{\dot{\mu}_{1}}{2 \alpha_{1}}$,

it results that

$\ddot{x}^{1}=\frac{k_{1} \beta_{3}}{2 \alpha_{1}} x^{1}+\frac{\beta_{1}\left(1-k_{1}\right)}{2 \alpha_{1}}$

which is a linear nonhomogeneous second order differential equation. From the linear homogeneous differential equation

$\ddot{x}^{1}-\frac{k_{1} \beta_{3}}{2 \alpha_{1}} x^{1}=0$ and from the characteristic equation

$\lambda^{2}-\frac{k_{1} \beta_{3}}{2 \alpha_{1}}=0$, with solutions, $\lambda_{1,2}= \pm \sqrt{\frac{k_{1} \beta_{3}}{2 \alpha_{1}}}$

the general solution of the homogeneous differential equation can be obtained:

$x^{1}(t)=a_{1} e^{\sqrt{\frac{k_{1} \beta_{3}}{2 \alpha_{1}}} t}+a_{2} e^{-\sqrt{\frac{k_{1} \beta_{3}}{2 \alpha_{1}}} t}$.

Hence, the general solution of the nonhomogeneous equation (9) is given by

$x^{\mathrm{l}}(t)=a_{1} e^{\sqrt{\frac{k_{1} \beta_{3}}{2 \alpha_{1}}} t}+a_{2} e^{-\sqrt{\frac{k_{1} \beta_{3}}{2 \alpha_{1}}} t}-\frac{\beta_{1}\left(1-k_{1}\right)}{k_{1} \beta_{3}}$

Using the initial conditions $x^{1}(0)=0, x^{1}(T)=s_{1}$ the following system is obtained:

$\left\{\begin{array}{l}a_{1}+a_{2}=\frac{\beta_{1}\left(1-k_{1}\right)}{k_{1} \beta_{3}} \\ a_{1} d_{1}+\frac{a_{2}}{d_{1}}=\frac{\beta_{1}\left(1-k_{1}\right)}{k_{1} \beta_{3}}+s_{1}\end{array}\right.$

where $d_{1}=e^{\sqrt{\frac{k_{1} \beta_{3}}{2 \alpha_{1}}} T}>1$ with the solution

$a_{1}=\frac{1-k_{1}}{d_{1}+1} \frac{\beta_{1}}{k_{1} \beta_{3}}+\frac{d_{1} s_{1}}{d_{1}^{2}-1}$

$a_{2}=\frac{d_{1}}{d_{1}+1} \frac{\beta_{1}\left(1-k_{1}\right)}{k_{1} \beta_{3}}-\frac{d_{1} s_{1}}{d_{1}^{2}-1}$

In the same way, using $\dot{x}^{2}=\frac{\mu_{2}}{2 \alpha_{2}}$,

$x^{2}(t)=b_{1} e^{\sqrt{\frac{k_{2} \beta_{3}}{2 a_{2}}} t}+b_{2} e^{-\sqrt{\frac{k_{2} \beta_{3}}{2 \alpha_{2}}} t}-\frac{\beta_{2}\left(1-k_{2}\right)}{k_{2} \beta_{3}}$

is obtained, where

$b_{1}=\frac{1-k_{2}}{d_{2}+1} \frac{\beta_{2}}{k_{2} \beta_{3}}+\frac{d_{2} s_{2}}{d_{2}^{2}-1}$

$b_{2}=\frac{d_{2}}{d_{2}+1} \frac{\beta_{2}\left(1-k_{2}\right)}{k_{2} \beta_{3}}-\frac{d_{2} s_{2}}{d_{2}^{2}-1}$

$d_{2}=e^{\sqrt{\frac{k_{2} \beta_{3}}{2 \alpha_{2}}} T}>1$

Finally, following equation is obtained

$x^{3}(t)=\frac{k_{1}\left(x^{1}\right)^{2}(t)+k_{2}\left(x^{2}\right)^{2}(t)}{2}$ 
Moreover, by introducing the expression (10) into the equation $\dot{\mu}_{1}=\beta_{1}\left(1-k_{1}\right)+x^{1} k_{1} \beta_{3}$ the following equation is obtained

$\dot{\mu}_{1}(t)=k_{1} \beta_{3} a_{1} e^{\sqrt{\frac{k_{1} \beta_{3}}{2 \alpha_{1}}} t}+k_{1} \beta_{3} a_{2} e^{-\sqrt{\frac{k_{1} \beta_{3}}{2 \alpha_{1}}} t}$

which leads to

$\mu_{1}(t)=\sqrt{2 \alpha_{1} k_{1} \beta_{3}}\left(a_{1} e^{\sqrt{\frac{k_{1} \beta_{3}}{2 \alpha_{1}}} t}-a_{2} e^{-\sqrt{\frac{k_{1} \beta_{3}}{2 \alpha_{1}}} t}\right)+c_{1}$

Also, by using the same computation it results that $\mu_{2}(t)=\sqrt{2 \alpha_{2} k_{2} \beta_{3}}\left(b_{1} e^{\sqrt{\frac{k_{2} \beta_{3}}{2 \alpha_{2}}} t}-b_{2} e^{-\sqrt{\frac{k_{2} \beta_{3}}{2 \alpha_{2}}} t}\right)+c_{2}$

and the following control variables are obtained: $u^{1}=\frac{\mu_{1}}{2 \alpha_{1}}=\sqrt{\frac{k_{1} \beta_{3}}{2 \alpha_{1}}}\left(a_{1} e^{\sqrt{\frac{k_{1} \beta_{3}}{2 \alpha_{1}}} t}-a_{2} e^{-\sqrt{\frac{k_{1} \beta_{3}}{2 \alpha_{1}} t}}\right)+\frac{c_{1}}{2 \alpha_{1}}$ $u^{2}=\frac{\mu_{2}}{2 \alpha_{2}}=\sqrt{\frac{k_{2} \beta_{3}}{2 \alpha_{2}}}\left(b_{1} e^{\sqrt{\frac{k_{2} \beta_{3}}{2 \alpha_{2}}} t}-b_{2} e^{-\sqrt{\frac{k_{2} \beta_{3}}{2 \alpha_{2}}} t}\right)+\frac{c_{2}}{2 \alpha_{2}}$

Moreover, from the relation

$x^{1}(T)-x^{1}(0)=\int_{0}^{T} \dot{x}^{1}(t) d t=\int_{0}^{T} u^{1}(t) d t$

the following equation is obtained:

$S_{1}=\int_{0}^{T}\left(\sqrt{\frac{k_{1} \beta_{3}}{2 \alpha_{1}}}\left(a_{1} e^{\sqrt{\frac{k_{1} \beta_{3}}{2 \alpha_{1}}} t}-a_{2} e^{-\sqrt{\frac{k_{1} \beta_{3}}{2 \alpha_{1}}} t}\right)+\frac{c_{1}}{2 \alpha_{1}}\right) d t$

which leads to

$s_{1}=a_{1}\left(e^{\sqrt{\frac{k_{1} \beta_{3}}{2 \alpha_{1}}} T}-1\right)+a_{2}\left(e^{-\sqrt{\frac{k_{1} \beta_{3}}{2 \alpha_{1}}} T}-1\right)+\frac{c_{1}}{2 \alpha_{1}} T$

and it results that

$c_{1}=\frac{2 \alpha_{1}}{T}\left(s_{1}-a_{1}\left(e^{\sqrt{\frac{k_{1} \beta_{3}}{2 a_{1}}} T}-1\right)-a_{2}\left(e^{-\sqrt{\frac{k_{1} \beta_{3}}{2 \alpha_{1}}} T}-1\right)\right)$

In the same way, the following can be obtained:

$c_{2}=\frac{2 \alpha_{2}}{T}\left(s_{2}-b_{1}\left(e^{\sqrt{\frac{k_{2} \beta_{3}}{2 \alpha_{2}}} T}-1\right)-b_{2}\left(e^{-\sqrt{\frac{k_{2} \beta_{3}}{2 \alpha_{2}}} T}-1\right)\right)$
Finally, the following control variables are obtained:

$u^{1}(t)=\sqrt{\frac{k_{1} \beta_{3}}{2 \alpha_{1}}}\left(a_{1} e^{\sqrt{\frac{k_{1} \beta_{3}}{2 \alpha_{1}}} t}-a_{2} e^{-\sqrt{\frac{k_{1} \beta_{3}}{2 \alpha_{1}}} t}\right)$

$+\frac{1}{T}\left(s_{1}-a_{1}\left(e^{\sqrt{\frac{k_{1} \beta_{3}}{2 \alpha_{1}}} T}-1\right)-a_{2}\left(e^{-\sqrt{\frac{k_{1} \beta_{3}}{2 \alpha_{1}}} T}-1\right)\right)$

$u^{2}(t)=\sqrt{\frac{k_{2} \beta_{3}}{2 \alpha_{2}}}\left(b_{1} e^{\sqrt{\frac{k_{2} \beta_{3}}{2 \alpha_{2}}} t}-b_{2} e^{-\sqrt{\frac{k_{2} \beta_{3}}{2 \alpha_{2}}} t}\right)$

$+\frac{1}{T}\left(s_{2}-b_{1}\left(e^{\sqrt{\frac{k_{2} \beta_{3}}{2 \alpha_{2}}} T}-1\right)-b_{2}\left(e^{-\sqrt{\frac{k_{2} \beta_{3}}{2 \alpha_{2}}} T}-1\right)\right)$

The solution above is optimal because the Hamiltonian function is convex.

Next, the following numerical example will be considered:

$s_{1}=2, s_{2}=2, s_{3}=2, \beta_{1}=4, \beta_{2}=4, \beta_{3}=2$,

$\alpha_{1}=2, \alpha_{2}=2, k_{1}=0.5, k_{2}=0.5, T=2$

and by using (10), (11), (12) the optimal solution is obtained, as illustrated in Figure 1.

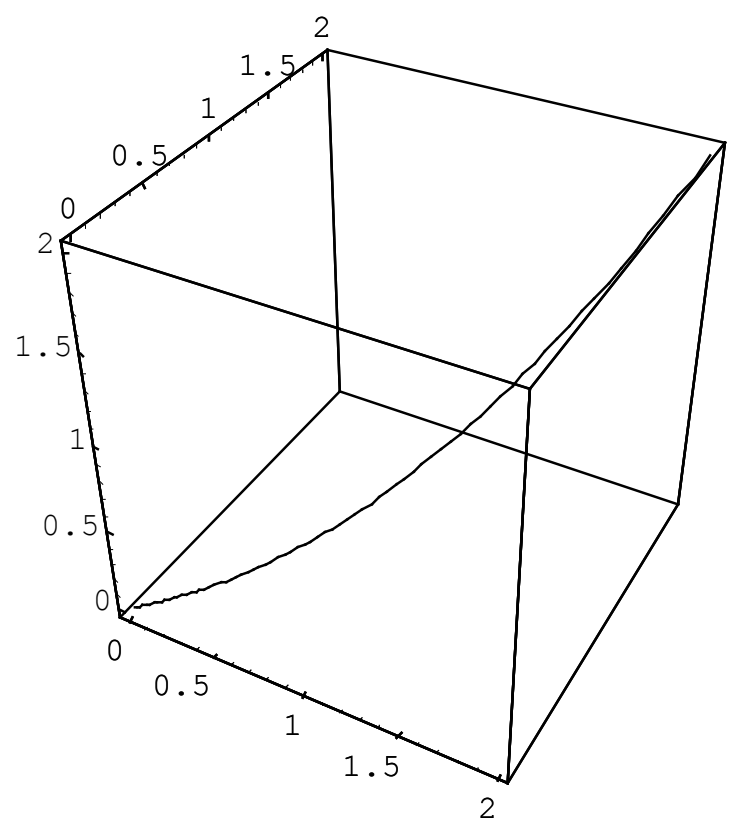

Figure 1. The optimal solution for the numerical example

For this example the control variables $u^{1}(t)=u^{2}(t)$ are obtained and are represented for $t \in[0,2]$ in Figure 2. 


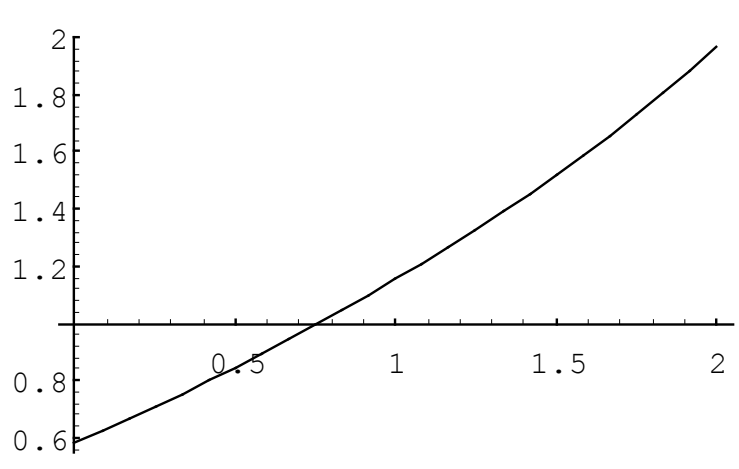

Figure 2. The control variables for $t \in[0,2]$

Figure 3 is obtained for the control variables in the case $t \in[0,10]$.

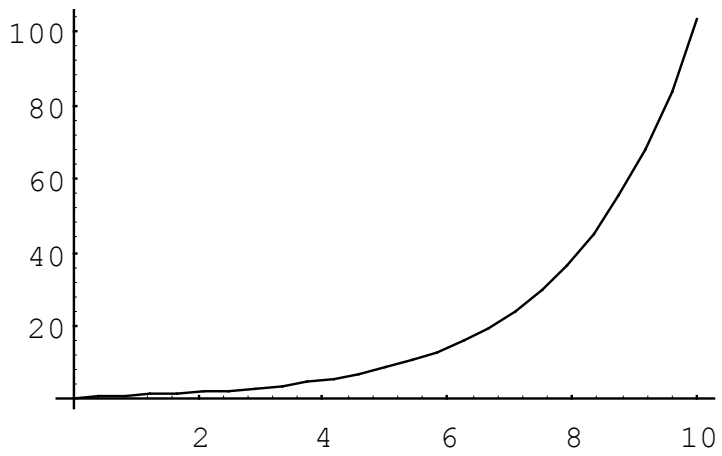

Figure 3. The control variables for $t \in[0,10]$

\section{REFERENCES}

1. Arrow, K. J. (1968). Applications of Control Theory to Economic Growth, Mathematics of the Decision Sciences, part 2. American Mathematical Society.

2. Brocket, R. W. (1973). Lie Algebra and Lie Groups in Control Theory, Geometric Methods in System Theory, 43-82. Springer.

3. Caputo, M. (2005). Foundations of Dynamic Economic Analysis: Optimal Control Theory and Applications. Cambridge University Press.

4. Chen, Z. (2018). Optimization of production inventory with pricing and promotion effort for a single-vendor multi-buyer system of perishable products, International Journal of Production Economics, 203, 333-349.

5. Dolgui A., Ben Ammar, O., Hnaein, F. \& Louly, M. (2013). A State of the Art on Supply Planning and Inventory Control under Lead Time Uncertainty, Studies in Informatics and Control, 22(3), 255-268, DOI: 10.24846/ v22i3y 201302

\section{Conclusion}

In this paper, a problem of inventory and production was solved by using the optimal control techniques for driftless control affine systems. Apart from its economic applications, the novelty of this paper consists in the application of the Pontryagin Maximum Principle at the level of a new framework, called the Lie algebroid, which in this case is an integrable distribution of the tangent space. Moreover, the controllability of the above-mentioned systems is solved by using the properties of the Lie brackets for the vector fields of distribution and involves restrictions on the final quantities required. Finally, a numerical example is given.

6. Gayon, J. P., Vercraene, S. \& Flapper, S. D. (2017). Optimal Control of a ProductionInventory System with Product Returns and two Disposal Options, European Journal of Operational Research, 262(2), 499-508.

7. Isidori, A. (1995). Nonlinear Control Systems. Springer-Verlag.

8. Kamien, M. I. \& Schwartz, N. L. (2006). Dynamic Optimization. Elsevier.

9. Khan, Q., Akmeliawati R. \& Khan M. A. (2018). An Integral Sliding Mode-Based Robust Consensus Control Protocol Design for Electro-Mechanical Systems, Studies in Informatics and Control, 27(2), 147-154, DOI: $10.24846 / \mathrm{v} 27 \mathrm{i} 2 \mathrm{y} 201802$

10. LaValle, S. M. (2006). Planning Algorithms. Cambridge University Press.

11. Li, M. \& Wang, Z. (2017). An integrated replenishment and production control policy under inventory inaccuracy and time-delay, Computers \& Operations Research, 88, 137-149. 
12. Mackenzie, K. (1987). Lie Groupoids and Lie Algebroids in Differential Geometry, London Mathematical Society, Lecture Note Series 124. Cambridge University Press.

13. Martinez, E. (2004). Reduction in Optimal Control Theory, Reports on Mathematical Physics, 53(1), 79-90.

14. Ortega, M. \& Lin, L. (2004). Control Theory Applications to the Production-Inventory Problem: a Review, International Journal of Production Research, 42(11), 2303-2322.

15. Pontryagin, L. S., Boltyanskii, V. G., Gamkrelidze, R. V. \& Mishchenko, E. F. (1962). The Mathematical Theory of Optimal Processes. John Wiley \& Sons.

16. Popescu, L. (2009). Lie Algebroids Framework for Distributional Systems, Annals of Alexandru Ioan Cuza University, Iasi, series I, Mathematics, 55(2), 257-274.
17. Popescu, L. (2017). Symmetries of Second Order Differential Equations on Lie Algebroids, Journal of Geometry and Physics, 117, 84-98.

18. Popescu, L. (2018). Mechanical Structures on Lie Algebroids, Mediterranean Journal of Mathematics, 15:154.

19. Seierstad, A. \& Sydsater, K. (1987). Optimal Control Theory with Economic Applications. North-Holland, NL.

20. Sethi, S.P.\& Thompson, G. L.(2000). Optimal Control Theory: Applications to Management Science and Economics. Springer.

21. Weber, T. A. (2011). Optimal Control Theory with Applications in Economics. MIT Press. 